> La sollicitation des patients pour participer à des recherches dans un contexte hospitalier est en augmentation constante, notamment en cancérologie, et encouragée par le Plan cancer. Nous présentons ici une réflexion, fondée sur la littérature scientifique, qui interroge le rapport des patients à la présence de la recherche au sein de la structure de soins qui les accueille. Nous nous focalisons plus particulièrement sur le consentement à participer à une tumorothèque. Cette approche est illustrée par les résultats d'une étude menée auprès de patients traités dans un centre de lutte contre le cancer. En l'état actuel des connaissances, il se dégage une opinion globalement favorable à la présence d'activités de recherche biomédicale en lien avec l'activité clinique, et même un souhait exprimé de sollicitations à participer davantage à ces recherches au côté des équipes scientifiques. <

\section{Le contexte}

Le contexte actuel des recherches internationales qui s'appuient sur l'analyse à grande échelle des tumeurs, dont la collecte et la conservation sont organisées au sein d'infrastructures dénommées tumorothèques, et sur la nécessité de définition de nouveaux traitements ciblés sur le profil génomique de ces tumeurs, soulève une question centrale, celle du point de vue des patients pris en charge. Cela concerne d'une part la perception et le vécu des personnes dont on sollicite la participation à des recherches, et d'autre part la nature du consentement et les modalités de son recueil, que ce soit dans le cadre d'une participation à un essai clinique ou à une tumorothèque, notamment si un transfert international des données cliniques et des échantillons prélevés est envisagé.

Depuis leur création en 1945, les Centres de lutte contre le cancer (CLCC), comme les Centres hospitaliers universitaires, ont vocation à assurer une triple mission de soins, d'enseignement et de recherche ${ }^{1}$. Si l'activité de

${ }^{1}$ www.unicancer.fr/le-groupe-unicancer/les-missions

La recherche biomédicale à l'ère des tumorothèques

Point de vue des patients atteints de cancer

Julien Mancini ${ }^{1,2,5}$, Christian Chabannon ${ }^{3,4}$, Norbert Vey ${ }^{3,4}$, Lise-Marie Billard-Daufresne ${ }^{3,4}$, Isabelle Pellegrini ${ }^{1,5}$, Claire Julian-Reynier ${ }^{1,3,5}$

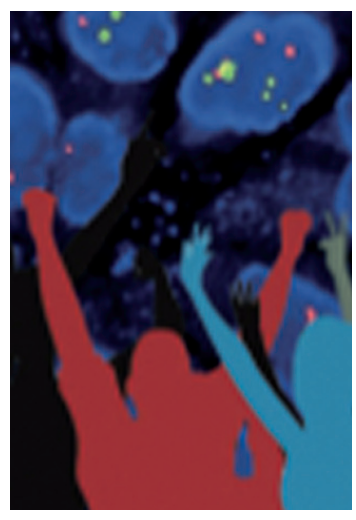

recherche a toujours été présente et souvent multicentrique du fait de la mise en réseau des institutions, cette activité s'est notablement développée au point de changer d'échelle au cours des dernières années. La recherche en oncologie est évolutive et revêt différentes formes, et les travaux conduits par les expérimentateurs doivent s'inscrire dans l'une des différentes catégories définies par les réglementations. Ainsi en France, la loi distingue la recherche biomédicale - qui porte sur le malade -, des recherches scientifiques et génétiques - qui utilisent des éléments dérivés du corps humain avec la finalité de produire des informations de nature non génétique ou génétique respectivement. La création des tumorothèques a introduit, dans certains contextes, une sollicitation systématique des patients. Un consentement leur est alors expressément demandé pour l'utilisation à des fins de recherche des échantillons biologiques qui ont été prélevés au cours des soins [1]. À la sollicitation en routine pour participer à des «biobanques » viennent s'ajouter les éventuelles propositions de participer à des essais cliniques. Ces dernières sollicitations sont globalement considérées comme trop rares, notamment par l'Institut national du cancer, compte tenu des besoins actuels de définition de nouvelles prises en charge efficaces, mais elles devraient augmenter au vu des mesures du plan Cancer 2009-2013 (voir mesure 4.2 : augmenter l'inclusion dans les essais cliniques en cancérologie $e^{2}$ ).

2 http://www.plan-cancer.gouv.fr/le-plan-cancer/5-axes-30-mesures/axe-recherche/mesure-4/ actions.html 
Parallèlement, de grands essais multicentriques internationaux (voir mesure 4.6 : développer la coopération internationale dans les essais cliniques $^{1}$ ), qui nécessitent le transfert d'échantillons biologiques avec les données cliniques, soulèvent des interrogations quant à la circulation des données clinicobiologiques à une grande échelle. Le Plan cancer précise notamment que l'effort de constitution de tumorothèques doit être poursuivi et s'articuler avec les initiatives des autres pays européens. Les transferts de prélèvements devraient donc croître, facilités par la constitution de tumorothèques virtuelles et la publication de catalogues de collections d'échantillons [2, 3].

\section{Perception par la population et les patients de l'existence d'une activité de recherche aux côtés de l'activité clinique}

Globalement, la population française accorde une grande importance à la recherche médicale (Tableau I). Des données recueillies auprès de 574 patients pris en charge dans un CLCC montrent que, à la suite de leur expérience personnelle, les patients sont convaincus de la qualité de cette recherche médicale française [4]. Une étude américaine auprès de malades chroniques montre qu'il existe une confiance globale envers les médecins chercheurs [5], confirmée par nos données [4]. Les patients expriment des attentes particulièrement élevées et déplorent l'insuffisance des financements alloués.

Dans notre expérience, l'existence d'activités de recherche aux côtés des activités purement cliniques était souvent connue des patients avant leur venue dans le CLCC ; sinon, elle a été rapidement identifiée par les patients et vécue positivement. Les trois-quarts des patients de notre enquête déclaraient que l'existence d'une activité de recherche est « assurément un critère de qualité pour les soins » [4] et, parmi les dix-neuf patients qui ont eu un entretien de recherche approfondi [6], cette perception s'exprime par exemple dans cet extrait d'entretien : " J'avais entendu parler de I'IPC, oui surtout dans la recherche. J'en avais entendu parler pas en tant qu'hôpital ; dans la recherche. Donc l'Institut Paoli-Calmettes, institut de recherche. Ce qui est un gage de sérieux, absolument. C'est sûr, je savais qu'il y avait quand même un accueil des malades, et puis bien sûr, un hôpital de jour et un hôpital de nuit, mais surtout institut de recherche (ton insistant). »(Augustin, 59 ans, cancer colorectal), ou dans celui-ci : "Je suis traité dans le cadre d'un protocole, là. J'ai signé un protocole oui, un suivi médical vis-à-vis du cancer du foie. Il y a aussi des assemblées de médecins qui se tiennent, qui se confrontent. C'est bien ça. Effectivement dans ce genre de maladie on imagine mal le médecin qui reste isolé. Ce n'est pas possible. Dieu merci, ça avance très vite, la recherche, et encore plus dans le domaine du cancer. » (Jules, 69 ans, cancer colorectal).

Les essais cliniques: souhait de propositions et d'implication dans la prise de décision

Dans le domaine des essais cliniques, de nombreux travaux se sont focalisés sur les attitudes des patients et les raisons individuelles qui les conduisent à participer - ou refuser de participer - à un essai [7]. Même si le recours à la randomisation nécessite une information approfondie, la majorité des patients atteints de cancer sont réceptifs à la proposition de participation à un essai clinique [8] et apprécient la prise en charge structurée proposée dans le cadre de tels essais thérapeutiques [9]. Ainsi, pour améliorer les taux d'inclusion, les recherches doivent s'intéresser non seulement aux refus des patients mais également aux raisons pour lesquelles les médecins ne proposent pas systémati-

\begin{tabular}{|c|c|c|}
\hline & $\begin{array}{c}\text { Patients } \\
\text { suivis à I'IPC }\end{array}$ & $\begin{array}{l}\text { Population } \\
\text { française }^{2}\end{array}$ \\
\hline La recherche médicale en France est essentielle pour l'avenir de notre santé & $97,4 \%$ & $97 \%$ \\
\hline La recherche médicale est un enjeu médical important pour la France & $92,0 \%$ & $81 \%$ \\
\hline La recherche médicale en France est performante & $90,6 \%$ & $78 \%$ \\
\hline \multirow[t]{2}{*}{ La recherche médicale en France dispose de crédits et financements suffisants } & $19,5 \%$ & $25 \%$ \\
\hline & $\begin{array}{c}\text { Patients } \\
\text { suivis à I'IPC }\end{array}$ & $\begin{array}{l}\text { Population } \\
\text { américaine }^{3}\end{array}$ \\
\hline Score de confiance envers les médecins chercheurs $(4-20)$, moyenne \pm écart type & $15,6 \pm 2,4$ & $12,4 \pm 2,7$ \\
\hline
\end{tabular}

Tableau I. Comparaison des réponses obtenues auprès de patients d'un centre de lutte contre le cancer avec les données de la littérature.

${ }^{1}$ Données issues de notre enquête auprès de 574 patients traités à I'Institut Paoli-Calmettes (IPC) [4].

${ }^{2}$ Sondage pour les 60 ans de la Fondation pour la recherche médicale auprès d'un échantillon représentatif de 1000 français (TNS direct, 10/09/2007, http://www.frm.org/nos-dossiers/videos/les-francais-et-la-recherche-medicale-3.htm).

${ }^{3}$ Données issues d'une enquête auprès de 3623 patients asthmatiques ou diabétiques [5]. 
quement à tous leurs patients éligibles de participer [10]. Si la possible influence des médecins sur les décisions de leurs patients a été assez peu étudiée, une étude qualitative montre une autonomie préservée des patients face à la décision de participer à un essai thérapeutique [11]. Dans l'étude réalisée à l'Institut Paoli-Calmettes (IPC, CLCC de Marseille) [4, 6], 7 \% des patients déclaraient avoir été sollicités pour participer à un essai clinique; ces sollicitations étaient perçues comme insuffisamment fréquentes dans $23 \%$ et trop fréquentes dans seulement $8 \%$ des cas.

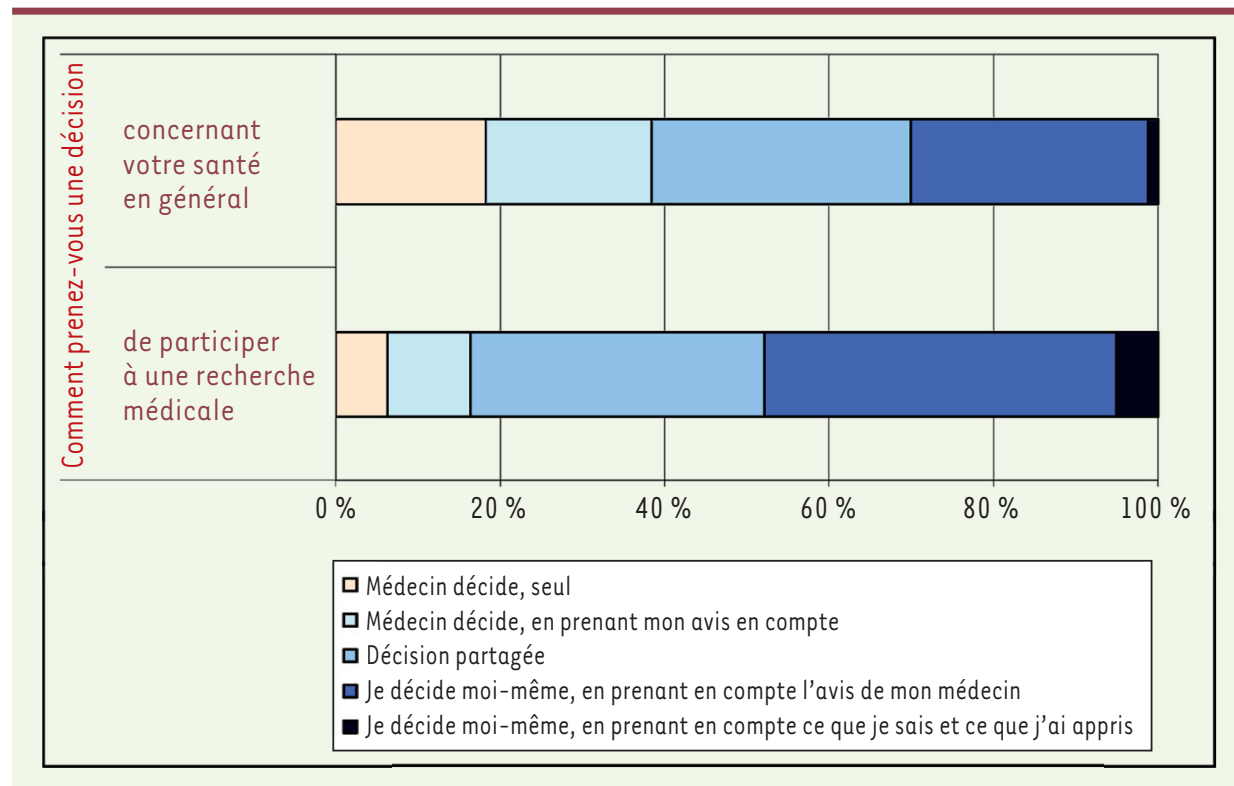

Figure 1. Implication dans la prise de décision selon le contexte décisionnel soins/recherche.

Ce sentiment s'exprime dans

le commentaire suivant: «Non, on ne m'a pas proposé de participer à des protocoles cliniques. Mais si on me dit qu'il y a un médicament à essayer, qui est efficace, je suis $100 \%$ d'accord hein. Il faut mettre toutes les chances de son côté hein ! Et puis faut faire avancer la science aussi.» (Cyprien, 54 ans, cancer colorectal).

Le fait de ne pas souhaiter être sollicité pour participer à un essai peut s'expliquer par la nécessité de décider soi-même de participer ou non, qui peut être considérée par certains patients comme une barrière [7]. Ainsi, plus de $10 \%$ des participants à un essai randomisé comparant deux chimiothérapies laisseraient leur médecin décider seul de leur participation à un nouvel essai hypothétique [12]. Nos résultats confirment que certains patients préfèrent laisser leur médecin prendre pour eux la décision de participer à une recherche, mais, globalement, les patients souhaitent s'impliquer personnellement de façon plus importante dans une décision qui concerne un protocole de recherche que dans le contexte d'un protocole de soins (Figure 1). En pratique, selon le type de recherche, les patients ne jugent pas toujours indispensable de s'impliquer dans la décision. En ce qui concerne les essais cliniques, le recueil d'un consentement signé n'est pas discutable et des initiatives se développent pour assister les patients dans cette prise de décision [13]. En ce qui concerne les tumorothèques, la nécessité du recueil d'un consentement volontaire n'est pas complètement acquise, et nous allons développer ce point.

\section{Le consentement pour la recherche à partir de prélèvements biologiques archivés dans une tumorothèque}

Lors de la mise en place des modalités des consentements requis pour que les chercheurs puissent utiliser un prélèvement tumoral conservé dans une tumorothèque, l'avis des patients a été sollicité dans plusieurs travaux. La majorité des individus dans la population générale
(66\%) est en faveur d'un recueil du consentement [14]. Cette proportion est similaire si l'on interroge les patients en cancérologie en France (61\%) [4]. Elle serait vraisemblablement plus élevée si les patients percevaient que les données cliniques, indirectement nominatives, sont transmises et que les échantillons prélevés sont susceptibles d'être utilisés pour des analyses génétiques identifiantes [15]. Les études qualitatives menées soulignent cette tendance favorable au recueil d'un consentement. Si certains patients peuvent craindre que le recueil des consentements puisse retarder la recherche [16], la plupart soulignent le caractère positif de cette procédure, signe du respect de l'autonomie des patients, acte important de solidarité et de réciprocité renforçant les liens avec les soignants [6, 17, 18].

Il est toutefois nécessaire de s'assurer de la qualité de la procédure. Des études, menées dans le contexte de biobanques destinées à la population générale ou à des patients diabétiques, ont mis en évidence la qualité insuffisante des consentements recueillis [19, 20]. En cancérologie, la période de l'annonce du diagnostic de cancer est peu propice à la transmission d'informations dans le cadre du recueil du consentement à participer à un essai clinique [21], et les documents d'information complets sont souvent longs et difficiles à comprendre [22]. Dans notre étude [4], $62 \%$ des patients se rappelaient correctement s'ils avaient, ou s'ils n'avaient pas, donné leur accord écrit. La difficulté de recueillir un tel consentement dans un laps de temps court par rapport à l'annonce du diagnostic est également mise 
en exergue par les entretiens individuels: «Pfff... Je ne me souviens plus. Je ne sais plus. Il me semble que j'ai lu ça, que j'ai dû dire oui. Ca me dit quelque chose, mais quand je suis arrivée ici, j'étais pas mal groggy, j'étais un peu perdue. Et on m'a fait tellement signer de papiers quand je suis arrivée! Donc, je ne peux plus vous dire exactement ce que j'ai signé. » (Jeannine, 73 ans, leucémie aiguë.)

Les débats persistent sur la nécessité de demander ou non un consentement et sur le caractère général ou spécifique de ce consentement dans le contexte des tumorothèques $[15,23,24]$. Avec le développement de tumorothèques virtuelles collaboratives, un nombre suffisant d'échantillons à analyser semble assuré mais un taux de consentement insuffisant peut entraîner une sélection non représentative des échantillons disponibles [25]. Toutefois, la signature du consentement garantit que l'information a bien été transmise au patient, ce qui est fréquemment nécessaire pour des collaborations internationales qui s'inscrivent dans un contexte réglementaire souvent compliqué par des réglementations différentes selon les pays [15].

\section{Les collaborations internationales \\ (et les contreparties financières)}

Néanmoins la démarche de recueil des consentements nécessite une organisation complexe associée à une mise « en quarantaine » d'échantillons initialement cryopréservés dans la tumorothèque, qui seront éventuellement détruits faute de consentement écrit donné par le patient pour autoriser la recherche. Le fonctionnement d'une tumorothèque proposant aux chercheurs des échantillons et des annotations cliniques de qualité nécessite des ressources importantes et l'utilité de la structure repose sur l'utilisation ultérieure des échantillons. Ainsi, les collaborations sont nécessaires et imposent des contreparties financières tout en s'inscrivant dans le cadre d'un véritable partenariat entre malades, médecins et chercheurs [26]. Il faut donc expliquer cette nécessité des transferts (internationaux) des échantillons (ou de leur analyse) et des données cliniques qui leur sont associées dans le cadre de recherches multicentriques, en insistant sur le respect de la confidentialité. En effet, seulement $35 \%$ des patients participant à une biobanque constituée pour des recherches génétiques savaient que d'autres personnes que leurs soignants pouvaient accéder à leurs données médicales [20] et moins de la moitié (42\%) des patients de notre enquête avaient réalisé que leur consentement incluait une autorisation d'accès au dossier médical, alors que celle-ci était explicitement mentionnée dans le document écrit [4]. D’autres études ont montré que le transfert à l'étranger des prélèvements n'était pas jugé problématique [27], mais il y avait une certaine réticence à faire confiance aux chercheurs non académiques [28] et accepter la «vente » d'un échantillon soulevait de grandes difficultés [24]. Notre étude confirme ces résultats de manière très nette [4]. Ainsi, si les patients suivis à I'IPC acceptaient en majorité le don d'un échantillon à un laboratoire privé ( $63 \%$, contre $82 \%$ pour un laboratoire public), la proportion qui acceptait une vente était très marginale, et ce quel que soit le type de laboratoire (13\% et $11 \%$ pour un laboratoire privé ou public respectivement).
Il faut donc clarifier la possibilité de transfert vers des laboratoires privés et systématiquement aborder les aspects financiers [29]. II faut distinguer la compensation financière correspondant aux frais de fonctionnement de l'infrastructure que constitue une biobanque appartenant au dispositif public de soins et de recherche, de la «vente d'échantillons » pouvant être associée à des bénéfices commerciaux. Les produits transfusionnels et médicaments dérivés du sang constituent un modèle économique dans un autre domaine d'activité médicale : le don de sang reste volontaire et non rémunéré, au moins dans un pays comme la France. Mais l'Établissement français du sang, établissement public en charge de la distribution de ces produits thérapeutiques, facture aux établissements de soins le coût de leur qualification et de leur préparation, sur la base de tarifs fixés annuellement par l'État. Les patients de notre enquête étaient convaincus du financement trop faible de la recherche en France [4]. Ils paraissaient prêts à accepter ces compensations indispensables au fonctionnement des infrastructures, si leur nécessité leur était expliquée et n'était pas associée à l'idée d'un bénéfice commercial démesuré comparé à leur propre niveau de vie [30].

\section{Conclusion}

Si la recherche en sciences humaines et sociales appliquée au cancer s'est développée sous l'impulsion des Plans cancer, relativement peu de travaux français et internationaux ont étudié la perception des activités de recherche par les patients atteints de cancer dans cette ère des tumorothèques. Cette brève revue de la littérature souligne la vision positive de la recherche biomédicale et de la présence d'une telle activité dans les structures d'accueil, perçue comme un indicateur de la qualité des soins proposés. Les patients jugent positivement les sollicitations proposées et sont prêts à s'investir personnellement dans ce type de décision afin de s'engager dans un véritable partenariat, comme illustre l'importance pour eux de signer activement le formulaire de consentement d'une tumorothèque et d'être sollicités pour éventuellement participer à des essais cliniques. Il faut toutefois s'assurer du libre arbitre des patients face à ces sollicitations et les consentements obtenus doivent refléter une adhésion éclairée. $\diamond$

\section{SUMMARY}

Perception by cancer patients of biomedical research in the era of large-scale biobanking

In French hospitals, patients are increasingly asked to participate in research, particularly in oncology where 
the development of research is stimulated at a national level (plan (ancer). This article express our thoughts based on the literature about the perception by cancer patients of research activities developed in the care centre where they are treated. We focus mainly on the consent for biobanking in a context in which cancer patients are routinely requested to donate tumour samples for research. This article presents the results of a survey among patients treated in a comprehensive cancer centre. The available literature shows that patients have an overall positive image of medical research and of the existence of research activities intertwined with medical care. Patients are globally expressing a wish for more proposals to participate in research in collaboration with scientific teams. $\diamond$

\section{CONFLIT D'INTÉRÊTS}

Les auteurs déclarent n'avoir aucun conflit d'intérêts concernant les données publiées dans cet article.

\section{RÉFÉRENCES}

1. Chabannon C, Lassailly F, Romain S, et al. Le réseau des centres de ressources biologiques (CRB) et tumorothèques de l'agglomération marseillaise. Med Sci (Paris) 2006 ; 22 (hors série $n^{\circ} 1$ ) : 26-31.

2. Chabannon C, Honstettre A, Daufresne LM, et al. La publication de catalogues de collections d'échantillons biologiques par les tumorothèques. Bull Cancer $2010 ; 97: 181-9$.

3. Reyrat $\varepsilon$, Geneve J. Constitution d'un catalogue commun a plusieurs CRLCC : un projet de la FNCLCC. Med Sci (Paris) 2006 ; 22 (hors série $n^{\circ} 1$ ) : 35-8.

4. Mancini J, Pellegrini I, Viret F, et al. Consent for biobanking: assessing the understanding and views of cancer patients. J Natl Cancer Inst 2011 ; 103 : 154-7.

5. Hall MA, Camacho F, Lawlor JS, et al. Measuring trust in medical researchers. Med Care 2006 ; $44: 1048-53$

6. Pellegrini I, Chabannon C, Mancini J, et al. Informed consent for biobanks and research: what make sense for cancer patients? Psychol Health $2009 ; 24: 307-8$.

7. Mills $\varepsilon$ J, Seely D, Rachlis B, et al. Barriers to participation in clinical trials of cancer: a metaanalysis and systematic review of patient-reported factors. Lancet Oncol $2006 ; 7: 141-8$.

8. Jenkins V, Farewell D, Batt L, et al. The attitudes of 1066 patients with cancer towards participation in randomised clinical trials. BrJ Cancer $2010 ; 103: 1801-7$.

9. Julian-Reynier C, Geneve J, Dalenc F, et al. Assessment of care by breast cancer patients participating or not participating in a randomized controlled trial: a report with the Patients' Committee for clinical trials of the Ligue nationale contre le cancer. J Clin Oncol 2007 ; $25: 3038-44$.

10. Minasian LM, O'Mara AM. Accrual to clinical trials: let's look at the physicians. J Natl Cancer Inst $2011 ; 103: 357-8$.

11. Ferguson PR. Patients' experiences and views of clinical trials. Med Law $2001 ; 20$ : 143-52.

12. Mancini J, Geneve J, Dalenc F, et al. Decision-making and breast cancer clinical trials: how experience challenges attitudes. Contemp Clin Trials 2007 ; 28 : 684-94.
13. Brown RF, Shuk $\varepsilon$, Leighl N, et al. Enhancing decision making about participation in cancer clinical trials: development of a question prompt list. Support Care Cancer $2010 ; 19$ : 1227-38.

14. Wendler $D$, Emanuel $\varepsilon$. The debate over research on stored biological samples: what do sources think? Arch Intern Med 2002 ; 162 : 1457-62.

15. Keogh B. European biobanks forge cross-border ties. J Natl Cancer Inst $2011 ; 103: 1429-31$.

16. Kaphingst KA, Janoff JM, Harris LN, Emmons KM. Views of female breast cancer patients who donated biologic samples regarding storage and use of samples for genetic research. Clin Genet 006 ; 69 : 393-8.

17. Axler RE, Irvine R, Lipworth W, et al. Why might people donate tissue for cancer research? Insights from organ/tissue/blood donation and clinical research. Pathobiology $2008 ; 75: 323-9$.

18. Allen J, McNamara B. Reconsidering the value of consent in biobank research. Bioethics $2011 ; 25: 155-66$.

19. Moutel G, de Montgolfier S, Meningaud JP, Herve C. Bio-libraries and DNA storage: assessment of patient perception of information. Med Law 2001 ; $20: 193-204$.

20. Ormond $K \varepsilon$, Cirino AL, Helenowski IB, et al. Assessing the understanding of biobank participants. Am J Med Genet 2009; 149A : 188-98.

21. Stevens T, Ahmedzai SH. Why do breast cancer patients decline entry into randomised trials and how do they feel about their decision later: a prospective, longitudinal, in-depth interview study. Patient Educ Couns $2004 ; 52: 341-8$.

22. Beardsley $\varepsilon$, Jefford M, Mileshkin L. Longer consent forms for clinical trials compromise patient understanding: so why are they lengthening? J Clin Oncol 2007 ; 25 : el3-4.

23. Stjernschantz Forsberg J, Hansson MG, Eriksson S. Biobank research: who benefits from individual consent? Br Med J 2011 ; 343 : d5647.

24. Vermeulen $\varepsilon$, Schmidt MK, Aaronson NK, et al. A trial of consent procedures for future research with clinically derived biological samples. Br J Cancer 2009 ; $101:$ : 1505-12.

25. Ransohoff DF, Gourlay ML. Sources of bias in specimens for research about molecular markers for cancer. J Clin Oncol $2010 ; 28$ : 698-704.

26. Maraninchi D. Valeurs et valorisation des tumorothèques. Med Sci (Paris) $2006 ; 22$ (hors série $n^{\circ} 1$ ) : 4 .

27. McMurter B, Parker L, Fraser RB, et al. Parental views on tissue banking in pediatric oncology patients. Pediatr Blood Cancer $2011 ; 57: 1217-21$.

28. Kaufman DJ, Murphy-Bollinger J, Scott J, Hudson KL. Public opinion about the importance of privacy in biobank research. Am J Hum Genet 2009; 85 : 643-54.

29. Watson RW, Kay EW, Smith D. Integrating biobanks: addressing the practical and ethical issues to deliver a valuable tool for cancer research. Nat Rev Cancer $2010 ; 10: 646-51$.

30. Skloot R. La vie immortelle d'Henrietta Lacks. Paris: Calmann-Lévy, 2011 : $440 \mathrm{p}$.
TIRÉS À PART

J. Mancini 